\title{
Visualization of Plasma Source Ion Implantation for Arrays of Multiple Targets
}

\author{
E. R. Keiter, J. H. Booske, and W. N. G. Hitchon
}

\begin{abstract}
Visualization of sheath overlap dynamics during plasma source ion implantation (PSII) of a multiple-target array is used to illustrate the effect of target spacing on dose uniformity over the surface of a single target. The simulations are conducted using a hybrid plasma model, and the images are created using the Tecplot package by Amtec. The simulations suggest scaling rules for sheath overlap time as a function of target spacing.
\end{abstract}

Index Terms - Ion implantation, PSII, pulsed sheaths.

$\mathbf{P}$ LASMA source ion implantation (PSII) has been extensively investigated as a cost-effective method for surface modification of nonplanar targets [1], as well as low-voltage implantation for ultrashallow doping of semiconductor [2]. Cost model analyses indicate that the economics of PSII favor large batch mode processing [3]. Hence, in order to achieve good spatial uniformity of dose, the process design parameters (plasma density, implantation voltage, target size, implantation pulse length, pulse duty cycle, and intertarget spacing) for a single batch should be chosen to ensure that any between target overlap of counter expanding sheaths is below a level at which significant dose reduction occurs due to ion density depletion. Prior experimental studies [4] indicate that spacing the targets such that no significant ion sheath overlap is expected during the implantation pulse results in dose uniformity over the surface of a single (spherical) target to within $+22 \%$.

Here we use visualization to help illustrate and quantify the phenomenon and effect of sheath overlap during PSII of an array of multiple targets. To investigate the sheath expansion dynamics, we use a numerical model consisting of a fourdimensional (two space dimensions, two velocity dimensions) convective scheme formulation for ion motion, coupled with a nonlinear Poisson solver which assumes Boltzmann electrons. The nonlinear Poisson solver is handled by SGFramework numerical software [5]. A full description of the model appears in [6].

We applied this model to several different configurations of targets, to investigate the effects of sheath overlap. A typical geometry can be observed in Fig. 1, in which electrostatic potentials around three circular targets are shown for three different times. The background gas is 5 mtorr nitrogen, and the implantation species is $\mathrm{N}_{2}^{+}$. The electron temperature is $2.0 \mathrm{eV}$. Initially a uniform plasma is assumed, with a plasma

Manuscript received July 13, 1998. This work was supported by the National Science Foundation (ECS-8721545).

E. R. Keiter was with the Department of Electrical and Computer Engineering, University of Illinois, Urbana, IL 61801-2991 USA. He is now with Sandia National Laboratories, Parallel Computational Sciences, Albuquerque, NM 87108 USA (e-mail: erkeite@landau.cs.sandia.gov).

J. H. Booske and W. N. G. Hitchon are with the Department of Electrical and Computer Engineering, University of Wisconsin, Madison, WI 53706 USA.

Publisher Item Identifier S 0093-3813(99)02566-7.
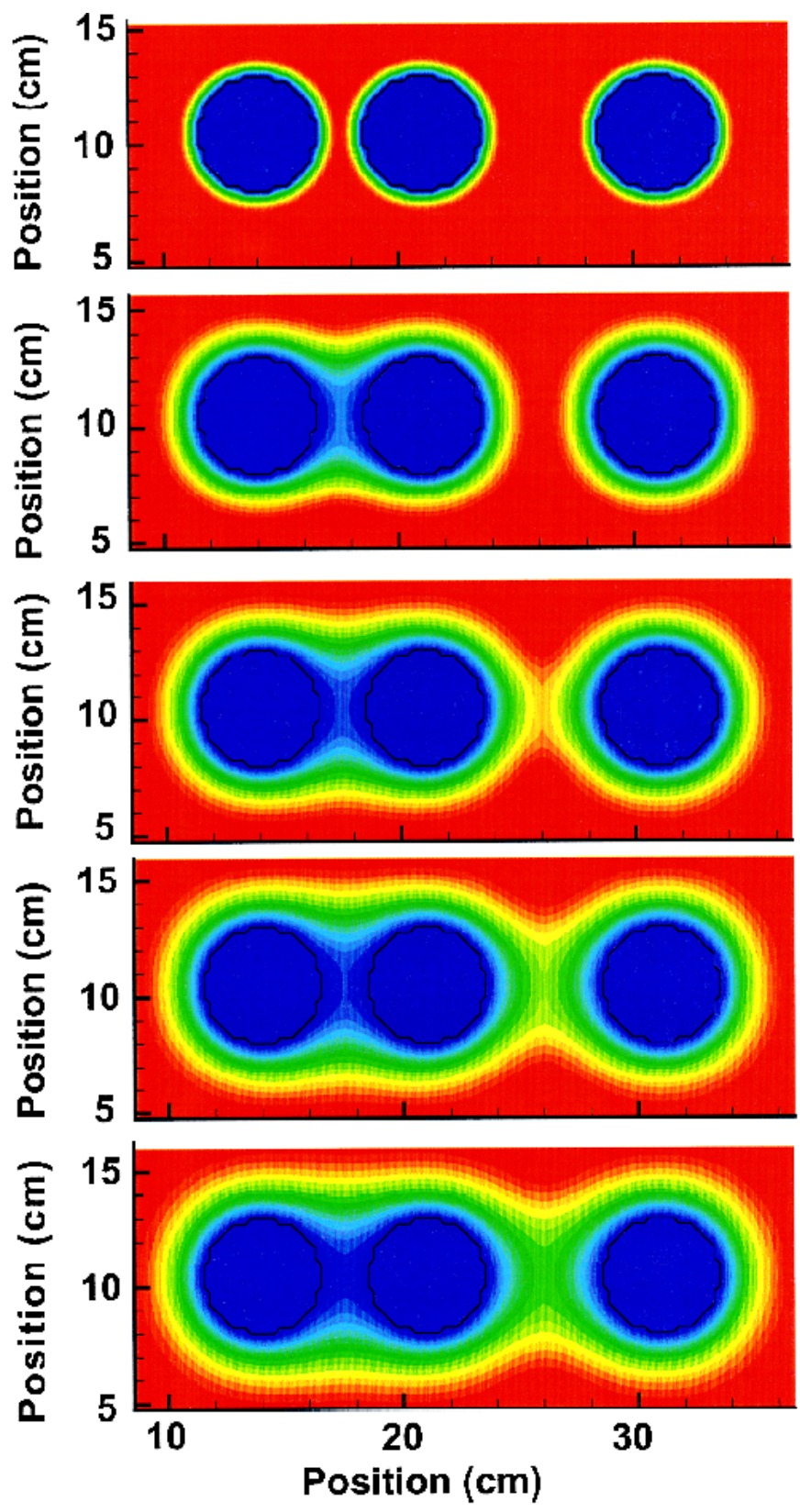

Fig. 1. Electrostatic potential at five different times: $5.0 \times 10^{-7}, 5.0 \times$ $10^{-6}, 1.0 \times 10^{-5}, 1.5 \times 10^{-5}$, and $2.0 \times 10^{-5} \mathrm{~s}$. The peak potential is $5 \mathrm{kV}$ in all five cases.

potential of $20 \mathrm{~V}$. The targets are biased with a ramping rate of $1.0 \times 10^{10} \mathrm{~V} / \mathrm{s}$, so that the targets have a bias of $5 \mathrm{kV}$ after $5.0 \times 10^{-7} \mathrm{~s}$. The target spacing between the two closest target centers is $7.0 \mathrm{~cm}$, while that of the two farthest targets is $10 \mathrm{~cm}$. The diameter of each target is $5.0 \mathrm{~cm}$. The expected final sheath width, given by the Child-Langmuir law, 


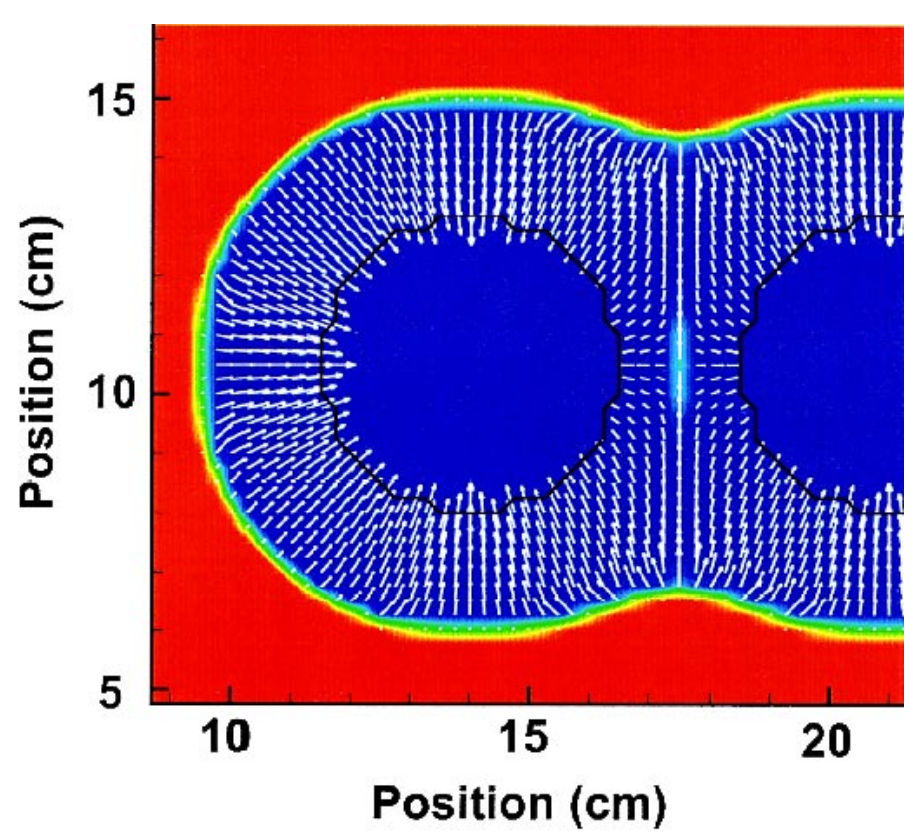

Fig. 2. Ion density and ion flux vectors for the left target. Peak ion density is $1.0 \times 10^{10}$.

is approximately $3.0 \mathrm{~cm}$, so sheath overlap should become a factor between all the pairs of neighbor targets at some point in the simulation.

The electrostatic potentials are shown for five separate times $\left(5.0 \times 10^{-7}, 5.0 \times 10^{-6}, 1.0 \times 10^{-5}, 1.5 \times 10^{-5}\right.$, and 2.0 $\left.\times 10^{-5} \mathrm{~s}\right)$ in Fig. 1. The first point in time corresponds to the end of the ramp-up phase, after which the bias on the target is constant. At this point in time, the sheaths of the nearest targets have yet to overlap. For the second case the sheaths between the closest two targets are overlapping and the sheaths between the other pair has just begun to intersect. The third case has significant overlap between all nearest neighbor targets.

The ion density and ion flux vectors are shown for the leftmost target in Fig. 2. For the interior surfaces, the incident ion flux is less than for the corresponding locations on the outside of the left target. Due to the potential saddle point between this target and its neighbor, there is a small buildup of ion density on the line of symmetry between the two targets. This effect could mitigate the effect of sheath overlap, as the ion flux will be somewhat larger. The difference between interior and exterior ion flux is proportional to the extent of the sheath overlap, which is in turn proportional to the target spacing. As the initial transient ramp-up phase can often represent the majority of the total implant, an optimal choice of target spacing would be one in which the neighboring sheaths would not significantly overlap until after the initial ramp-up phase.

\section{REFERENCES}

[1] J. R. Conrad, J. L. Radtke, R. A. Dodd, F. J. Worzala, and N. C. Tran, "Plasma source ion implantation technique for surface modification of materials," J. Appl. Phys., vol. 62, no. 11, pp. 4591-4596, Dec. 1987.

[2] P. K. Chu, S. Qin, C. Chan, N. W. Cheung, and L. Larson, "Plasma immersion ion implantation-A fledgling technique for semiconductor processing," Mater. Sci. Engineer., vol. 17, no. 6-7, pp. 207-277, Nov. 1996.

[3] T. S. Ebert, R. Stewart, F. Sainfort, and J. H. Booske, "A cost model of commercial plasma source ion implantation for corrosion protection of multiple objects via batch processing," Surf. Coatings Technol., vol. 102, pp. 8-18, 1998.

[4] J. R. Conrad, S. Baumann, R. Fleming, and G. P. Meeker, "Plasma source ion implantation dose uniformity of a 2 Y 2 array of spherical targets," J. Appl. Phys., vol. 65, no. 4, pp. 1707-1712, Feb. 1989.

[5] K. M. Kramer and W. N. G. Hitchon, Semiconductor Devices: A Simulation Approach. Englewood Cliffs, NJ: Prentice-Hall, 1998.

[6] E. R. Keiter, W. N. G. Hitchon, and M. J. Goeckner, "A kinetic model of pulsed sheaths," Phys. Plasmas, vol. 1, no. 11, pp. 3709-3712, Nov. 1994. 